\title{
SOME EFFECTS OF NITRATES IN BACTERIOLOGICAL MEDIA
}

\author{
BY \\ G. T. COOK AND R. KNOX \\ From the Public Health Laboratory, Oxford \\ (RECEIVED FOR PUBLICATION JUNE 9, 1950)
}

In the routine examination of faeces by means of the "screening" plate pre. viously described (Knox, 1949) it was found that on certain batches of the medium known gas-producing organisms failed to produce gas from the mannitol. Since this was the first time that the medium had failed in over a year's routine use it was felt that an explanation would be of some interest.

The explanation proved to be simple. It was recalled that a little earlier an error had been discovered in one stage of the bulk preparation of the laboratory tryptic digest broth ; after digestion nitric acid had been used instead of the usual hydrochloric acid. At the time the possible importance of this was not appreciated, but when this error was revealed and related to the odd failure of gas production on the screening plates, the true explanation was immediately suggested. It is known that bacterial gas production is inhibited by the addition of hydrogen acceptors such as nitrate (Pakes and Jollyman, 1901) or tetrathionate (Knox, Gell, and Pollock, 1943) to cultures or washed suspensions of gas-producing organisms which are capable of using these substrates instead of oxygen as alternative hydrogen acceptors. The exact mechanism is not quite clear, but it would appear that the formation of hydrogenlyase is suppressed in a specific and probably competitive way, since it is only with organisms capable of reducing these substrates that gas production is inhibited in their presence. But whatever the precise explanation the following evidence shows that nitrate in the screening plates was in fact acting as a powerful suppressor of gas formation.

\section{Suppressor Effects of Nitrate}

1. Samples of the defective medium were found to contain nitrate. It was calculated that the concentration of nitrate in the medium would be of the order of 0.02M.

2. Batches of medium with and without potassium nitrate $(0.02 \mathrm{M})$ were prepared and inoculated in the usual way with salmonella and coliform organisms. The nitrate plates showed good acid production from mannitol but no gas; the control plates showed normal production of both acid and gas.

\section{Other Effects of Nitrate}

$\mathbf{H}_{2}$ S Production.- It had been observed that on the defective batches of medium, in addition to gas production from mannitol, production of $\mathrm{H}_{2} \mathrm{~S}$ was almost or 
completely suppressed. This was also shown to be due to the nitrate in the medium : normal batches of medium $A$ of the screening plate inoculated with $\mathrm{H}_{2} \mathrm{~S}$ producing strains of salmonellae showed excellent $\mathrm{H}_{2} \mathrm{~S}$ production which was completely suppressed in similar plates containing $0.02 \mathrm{M}$ potassium nitrate. That this effect may be of some practical importance in routine work was shown by some experiments with Wilson and Blair's medium made with and without $0.02 \mathrm{M}$ potassium nitrate. The control plates showed typical blackening with the characteristic halo and sheen around salmonella colonies, whereas the plates containing nitrate showed green colonies without any blackening. It is clearly possible that the unsuspected presence of other hydrogen acceptors could account for the failures which occur from time to time with Wilson and Blair's medium.

The explanation of this interference with $\mathrm{H}_{2} \mathrm{~S}$ production is obscure, but again it may be due to competition between organic or inorganic sulphur compounds on the one hand and nitrate on the other for a common enzyme system.

Effect on Blood Plates. - It was observed about this time that some batches of blood agar plates showed patchy brown discoloration usually in the neighbourhood of bacterial colonies. These areas were shown to be related to the presence of nitrate in the medium. Blood agar plates with and without $0.02 \mathrm{M}$ potassium nitrate were inoculated with each of the following five cultures: Staphylococcus aureus, Staphylococcus albus, Corynebacterium diphtheriae gravis and mitis, and a starchfermenting gelatin-liquefying corynebacterium (Jebb, 1948). There was brown discoloration of the plate containing nitrate inoculated with each of the first four cultures, all nitrate-reducers. There was no discoloration of the nitrate plate inoculated with the last culture nor of any of the inoculated plates without nitrate. Starchfermenting gelatin-liquefying corynebacteria are known to be unable to reduce nitrate, so it seemed as though the brown discoloration was related not to the presence of nitrate but to its reduction to nitrite. This suggestion was confirmed by finding that the addition of potassium nitrite to blood agar plates gave a similar brown discoloration. These observations formed the basis of the test for nitrate reduction described in the accompanying paper (Cook, page 359).

\section{Discussion}

These observations are of some interest since they illustrate admirably the intimate relation which can exist between routine and research in a bacteriological laboratory or, in the words of the late Dr. Marjory Stephenson, the different "levels" of microbiology, and the kinds of way in which each can be a stimulus to the other. A mistake in the media room led to a puzzling problem which was only solved by the use of information derived from fundamental studies of bacterial metabolism ; the application of this led in turn to a new test useful in routine work.

\section{Summary}

The accidental use of nitric instead of hydrochloric acid in routine medium making led to some interesting results, since the presence of nitrate in the basal medium accounted for (1) failure of gas production from mannitol by known gasproducing organisms; (2) failure of $\mathrm{H}_{2} \mathrm{~S}$ production by known $\mathrm{H}_{2} \mathrm{~S}$ producers ; (3) failure of salmonella organisms to produce the characteristic blackening on Wilson 
and Blair's medium; and (4) a patchy brown discoloration on blood agar plates associated with oxidation of haemoglobin to methaemoglobin by nitrate-reducing organisms.

Our thanks are due to Mr. A. H. Tomlinson, M.A., for performing the chemical tests, and to Mr. A. Peacock for drawing attention to the accidental use of nitric acid in the medium.

\section{REFERENCES}

Jebb, W. H. H. (1948). J. Path. Bact., 60, 403.

Knox, R. (1949). Ibid., 61, 343.

Gell, P. G. H., and Pollock, M. R. (1943). J. Hyg., Camb., 43, 147.

Pakes, W. C. C., and Jollyman, W. H. (1901). Proc. chem. Soc. Lond., 17, 39. 